\title{
Successful Implementation of Outpatient Parenteral Antimicrobial Therapy at a Medical Respite Facility for Homeless Patients
}

\author{
Alison M. Beieler, PA-C, MPAS ${ }^{1 *}$, Timothy H. Dellit, MD ${ }^{1,2}$, Jeannie D. Chan, PharmD, MPH ${ }^{1,3}$, Shireesha Dhanireddy, MD ${ }^{1,2}$, \\ Leslie K. Enzian, $\mathrm{MD}^{4}$, Tamera J. Stone, RN${ }^{4}$, Edward Dwyer-O'Connor, RN ${ }^{4}$, John B. Lynch, MD, MPH ${ }^{1,2}$
}

\begin{abstract}
${ }^{1}$ Infectious Diseases, Harborview Medical Center, Seattle, Washington; ${ }^{2}$ Department of Allergy and Infectious Diseases, University of Washington,

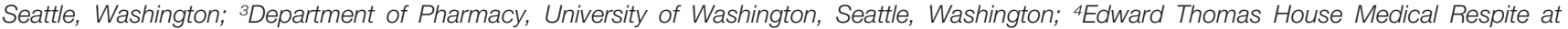
Jefferson Terrace, Harborview Medical Center, Seattle, Washington.
\end{abstract}

BACKGROUND: Outpatient parenteral antimicrobial therapy (OPAT) is a safe way to administer intravenous (IV) antimicrobial therapy to patients with the potential to decrease hospital length of stay (LOS). Often, homeless patients with complex infections, who could otherwise be treated as an outpatient, remain in the hospital for the duration of IV antibiotic treatment. Injection drug use (IDU) is a barrier to OPAT.

OBJECTIVE: To evaluate our experience with administering OPAT to homeless patients at a medical respite facility and determine if patients could complete a successful course of antibiotics.

DESIGN: Using retrospective chart review, demographics, diagnosis, and comorbidities including mental illness, current IDU, and remote IDU (>3 months ago) were recorded. Surgical, microbiologic, and antimicrobial therapy including route (IV or oral), duration of therapy, and adverse events were abstracted.
PARTICIPANTS: Homeless patients $>18$ years old who received OPAT at medical respite after discharge, no exclusions.

MAIN MEASUREMENTS: Primary outcome was successful completion of OPAT at medical respite. Secondary outcome was successful antimicrobial course completion for a specific diagnosis.

RESULTS: Forty-six (87\%) patients successfully completed a defined course of antibiotic therapy. Thirty-four (64\%) patients were successfully treated with OPAT at medical respite. Readmission rate was $30 \%$. The average length of OPAT was 22 days. The cost savings to our institution (using $\$ 1500 /$ day inpatient cost) was $\$ 25,000$ per episode of OPAT.

CONCLUSIONS: OPAT can be successful in a supervised medical respite setting for homeless patients with the help of a multidisciplinary team, and can decrease inpatient LOS resulting in cost savings. Journal of Hospital Medicine 2016;11:531-535. (C) 2016 Society of Hospital Medicine
Prolonged hospitalizations for complex patients with severe infections and difficult social situations are becoming very common in many institutions. Outpatient parenteral antimicrobial therapy (OPAT) is widely used ${ }^{1}$ and has been found to be a safe, efficient, and cost-effective way to administer intravenous (IV) antimicrobial therapy to patients, with the potential to decrease hospital length of stay (LOS) and to improve patient satisfaction. ${ }^{2}$ Infectious disease (ID) consultation should be involved to determine appropriate candidates for OPAT as well as a suitable drug regimen and duration of therapy, ${ }^{3}$ or if oral alternatives can be utilized. ${ }^{4}$ OPAT patients require close laboratory monitoring and provider follow-up for the duration of their care. The combination of ID consultation, patient selection, laboratory monitoring, and

\footnotetext{
*Address for correspondence and reprint requests: Alison Beieler, Harborview Infectious Disease Clinic, 325 9th Avenue, Box 359930 , Seattle, WA 98104; Telephone: 206-744-8215; Fax: 206-744-6564 E-mail: beielera@uw.edu

Additional Supporting Information may be found in the online version of this article.

Received: December 22, 2015; Revised: March 14, 2016; Accepted: March 31, 2016

2016 Society of Hospital Medicine DOI 10.1002/jhm.2597

Published online in Wiley Online Library (Wileyonlinelibrary.com).
}

follow-up care have been described as part of a proposed "OPAT bundle" in recent medical literature. ${ }^{5}$ Appropriate patient selection is a key component as to whether or not a patient will be successful with OPAT once discharged from the hospital. Current Infectious Diseases Society of America (IDSA) guidelines recommend that patients be evaluated for stable housing and ability to perform OPAT-specific duties prior to discharge. ${ }^{3}$

To our knowledge there are no published data regarding the use of OPAT at a medical respite facility for homeless patients with co-morbid substance abuse and mental illness issues. This may be due to perceived concerns of difficulty in administering OPAT to these disadvantaged patients for multiple reasons such as unstable or no housing, inability to stay engaged in medical care, and underlying mental illness and substance abuse problems. In particular, the concern for substance abuse, specifically injection drug use (IDU), is a significant problem. The current IDSA guidelines for OPAT recommend "patients who are likely to abuse a vascular access system are poor candidates for OPAT." 3

A major barrier to successful utilization of OPAT programs is the need for stable housing so that antibiotics can be administered in a safe setting. Recommending long-term parenteral therapy as an inpatient for all patients who are homeless or have a history of 
IDU can lead to prolonged hospitalizations, increased healthcare costs, and contribute to conflicts between patients and staff. Chemical dependence treatment is not available in most inpatient settings, leaving patients with addiction issues without options. Most patients would prefer, when given the choice, to be treated with OPAT outside of the inpatient setting. ${ }^{6}$

This study aimed to evaluate our experience with administering OPAT to homeless patients at a medical respite facility and to determine if patients could complete a successful treatment course of antibiotics for a variety of illnesses.

\section{METHODS}

Harborview Medical Center (HMC) is a 413-bed county hospital, and serves as a major teaching hospital for the University of Washington. It is a level 1 trauma/burn center for Washington, Wyoming, Alaska, Montana, and Idaho. The hospital has 61 psychiatric beds, 29 rehabilitation beds, and 89 intensive care unit beds, with over 60,000 emergency department visits per year. Harborview also serves as a public safety-net hospital for King County, providing \$219 million in charity care in 2013 .

Housed in a building adjacent to HMC is a 34-bed medical respite program, ${ }^{7}$ which was established in 2011 through collaboration with King County and 6 other hospitals to serve the homeless population needing medical care, similar to programs in Boston ${ }^{8}$ and San Francisco. ${ }^{9}$ It is staffed by a multidisciplinary team from HMC including a physician, nurse practitioners, registered nurses, medical assistants, mental health specialists, case managers, and security guards, and accepts patients from all hospitals and clinics within King County. To qualify for medical respite, patient must be homeless and require ongoing nursing needs (ie, wound care, parenteral therapy). Referred patients are screened by a nurse prior to admission. The projected daily cost at medical respite is $\$ 350$ per patient.

Medical respite is a harm-reduction model, which includes information on needle exchange programs, narcan kits and education on safer injection practices. Resources are available for patients wishing to start a rehabilitation program, including opiate replacement therapy. Patients may leave the premises during the day, but a curfew is enforced at 9 PM nightly. Patients sign a contract on admission to refrain from using their IV line for IDU and peripherally-inserted central catheter (PICC) port is secured and monitored for manipulation. Patients who exhibit threatening behavior or who use alcohol/drugs on site are discharged from the program. Patients in need of OPAT must keep nurse visits once or twice daily depending on medication and wound care. Medications needing more frequent dosing were placed on a battery-operated pump and changed once every 24 hours by nursing.

After obtaining approval from the University of Washington Institutional Review Board, we performed

\begin{tabular}{lc}
\multicolumn{1}{l}{ TABLE 1. Description of Patients Receiving } \\
Outpatient Parenteral Antimicrobial Therapy \\
$\begin{array}{l}\text { at Medical Respite } \\
\text { Comorbidities }\end{array}$ \\
\hline Hepatitis C infection & $32(60 \%)$ \\
Current IDU & $28(53 \%)$ \\
Psychiatric/mental illness & $14(26 \%)$ \\
Remote IDU & $9(17 \%)$ \\
Hypertension & $7(13 \%)$ \\
Diabetes type 1 or type 2 & $5(9 \%)$ \\
Rheumatologic diagnosis & $3(6 \%)$ \\
Obesity & $2(4 \%)$ \\
Cardiovascular disease & $2(4 \%)$ \\
Peripheral vascular disease & $2(4 \%)$ \\
Congestive heart failure & $2(4 \%)$ \\
Chronic kidney disease (any stage) & $1(2 \%)$ \\
HIV & $1(2 \%)$
\end{tabular}

NOTE: Abbreviations: HIV, human immunodeficiency virus; IDU, injection drug use.

a retrospective chart review of homeless patients over 18 years old discharged from HMC who received OPAT at medical respite from January 1, 2012 to January 1,2014 . There were no exclusions for race, gender, or insurance status. Patients included in the study were respite candidates, and required prolonged parenteral antibiotic therapy. Data collection was performed using a REDCap data collection tool and REDCap grant support. ${ }^{10}$ Demographics, diagnosis, and comorbidities, including mental illness, current IDU at time of admission, and remote IDU (last use $>3$ months ago) were obtained from the electronic medical record. Surgical, microbiologic, and antimicrobial therapy, including route (IV or oral), duration of therapy, and adverse events were abstracted. Primary outcome was defined as successful completion of OPAT at medical respite without nonadherence to therapy or readmission (for presumed OPAT failure). A secondary outcome was antimicrobial course completion for a specific diagnosis defined by achieving goal duration of parenteral and/or oral antibiotic therapy as deemed appropriate by an ID provider. Nonadherence is defined as missing greater than 2 doses of scheduled antibiotic, absence from respite for greater than 24 hours, evidence of line tampering, or expulsion from respite for violation of care agreement. Recurrence of infection was defined as subsequent infection at the same site, following completion of a prior antimicrobial course, at the most recent follow-up visit.

Continuous variables are expressed as the mean \pm standard deviation, and categorical variables are expressed as the proportion of the entire population. Categorical variables are compared using the $\chi^{2}$ test. A 2-sided $P$ value of $<0.05$ was considered statistically significant.

\section{RESULTS}

Fifty-one homeless patients were identified with 53 episodes of OPAT between January 1, 2012 and 


\begin{tabular}{|c|c|}
\hline & $\begin{array}{c}\text { No. of Episodes of Care, } \\
n=53(\%)\end{array}$ \\
\hline Successfully treated at medical respite & $34(64 \%)$ \\
\hline Nonadherent to therapy & $19(36 \%)$ \\
\hline Left respite with IV line in place & $\begin{array}{l}6 \text { [2 admitted, } \\
3 \text { orals, } 1 \text { lost] }\end{array}$ \\
\hline Missed IV doses and switched to orals & 5 \\
\hline Missed IV doses and admitted & 8 admitted \\
\hline Any hospital readmission & $16(30 \%)$ \\
\hline Readmissions, assumed "failures" & $10(19 \%)$ \\
\hline PICC-line-associated infection/bacteremia & 2 \\
\hline SIRS with suspected line infection & 2 \\
\hline Ongoing IDU /discharge from respite & 2 \\
\hline Nonadherent with OPAT/altercations & 3 \\
\hline Acute kidney injury & 1 \\
\hline Readmissions, not counted as "failures" & $6(11 \%)$ \\
\hline PICC malfunction (leaking) & $2[1$ had further OPAT $]$ \\
\hline Surgery & $4[3$ had further OPAT] \\
\hline
\end{tabular}

NOTE: Of the total readmissions, 5 completed IV therapy inpatient, 7 switched to orals. Abbreviations: IDU, injection drug use; IV, intravenous; OPAT, outpatient parenteral antimicrobial therapy; PICC, peripherally inserted central catheter; SIRS, systemic inflammatory response syndrome.

January 1, 2014. For ease of reporting, the number of episodes of OPAT $(\mathrm{n}=53)$ was used as the denominator instead of number of patients $(\mathrm{n}=51)$ for descriptive statistics. The average age was $45 \pm 10.4$ years (range, 22-62 years), $38(72 \%)$ patients were male, and $39(74 \%)$ were Caucasian. Comorbidities included $28(53 \%)$ patients with current IDU and 9 $(17 \%)$ with a remote history of IDU, $32(60 \%)$ with hepatitis C infection, and $14(26 \%)$ with mental illness (Table 1).

Forty-six $(87 \%)$ patients were evaluated by an ID physician during their admission. Diagnosis (some patients had multiple) requiring OPAT included: bacteremia in 28 , osteomyelitis in 22 , skin and soft tissue infection in 19, endocarditis in 15, and epidural abscess in 7 patients. Twenty-nine patients underwent surgical intervention. The pathogens recovered were primarily gram-positive organisms. Multidrug resistant organisms were isolated in 11 patients. The IV medications used included vancomycin, nafcillin, cefazolin, ertapenem, and daptomycin.

Forty-six $(87 \%)$ patients completed a defined course of antibiotic therapy (deemed appropriate therapy by an ID physician) for their specific infection. Thirty-four $(64 \%)$ patients were successfully treated with OPAT at medical respite. There were $19(36 \%)$ failures, which included nonadherent patients, some of whom required urgent readmission (Table 2). There were a total of 16 readmissions, and 10 of those were considered OPAT failures, whereas the other 6 were not (patients admitted for other reasons including, surgery, and IV malfunction). Of the total readmissions, 12 of those were current or remote IDU patients. There is a trend toward a higher prevalence of current/remote IDU among those with clinical failure $(15 / 19,79 \%)$ compared to those with clinical success $(22 / 34,65 \%)$ $(P=0.2788)$. Overall, $27(51 \%)$ patients were switched to oral therapy after completing an initial IV course. Oral agents used were: trimethoprimsulfamethoxazole, rifampin, doxycycline, fluconazole, linezolid, fluoroquinolones, and amoxicillin/clavulanic acid. The average length of OPAT was 22 days. The average daily cost of an acute-care bed day in 2015 was $\$ 1500$ at our institution. The cost savings to our institution (using $\$ 1500 /$ day inpatient cost compared to $\$ 350$ per day at medical respite) was $\$ 25,000$ per episode of OPAT.

During the course of OPAT, $7(13 \%)$ patients experienced an adverse event. Of those, we had 1 patient with drug rash, 1 with nausea, and 1 with diarrhea (not infectious). One patient developed leukopenia (white blood cells $<4.0$ ), and 2 patients developed neutropenia (absolute neutrophils $<750$ ). One patient developed significant elevation of creatinine $(>1.9 \times$ upper limit of normal) and required inpatient admission. An additional 5 patients had a small elevation of creatinine that did not meet the criteria listed above and were not counted as adverse events by definition. At the study conclusion, $36(68 \%)$ patients had no recurrence of infection at the most recent follow-up visit at HMC; length of follow-up ranged from 2 months to 2.5 years. One patient later died of nonOPAT-related complications. In total, 11 (21\%) patients were lost to follow-up, 1 with a peripherally inserted central catheter line in place.

\section{DISCUSSION}

We demonstrated that $87 \%$ of homeless patients were able to complete a defined course of antibiotic therapy, and $64 \%$ were successfully treated with OPAT at medical respite. To our knowledge this is the first study evaluating this specific population in which OPAT was received at medical respite. Our rate of adverse events (some that required change in drug therapy) was similar to other OPAT studies in the published literature, ranging from $3 \%$ to $10 \%$ in 1 study, ${ }^{3}$ and up to $11 \%$ in another. ${ }^{11}$ Our total readmission rate of $30 \%$ was similar to what current literature suggests, ranging from $9 \%{ }^{11}$ up to $26 \%{ }^{12}$ for OPAT patients. Notably, $11 \%$ of the readmissions were not related to OPAT failure. This supports the recommendation for close scrutiny of social behaviors in the OPAT patient-selection process; however, in certain circumstances, IDU alone may not be a reason to fully exclude someone from OPAT candidacy. Careful review of substance abuse history and evaluation of psychosocial factors, such as housing status, mental health history, and outpatient support system are needed. Furthermore, an evaluation of the patient's willingness to comply with care agreements while an inpatient and at respite, and ensuring 
appropriate resources for chemical dependency treatment are needed. Early consideration of oral antimicrobial options if the patient is readmitted for complications/nonadherence should be encouraged.

Our findings are consistent with results reported by Ho and colleagues, which demonstrated a success rate of $97 \%$ of IDU OPAT patients. ${ }^{13}$ They carefully chose 29 study patients from 906 in their OPAT program over several years, giving them daily infusions under close supervision. Patients signed an agreement to refrain from accessing their IV lines for drug use. Special security seals were used on all connections and tubing to prevent line tampering. Medical respite in King County uses a similar technique, using a Tegaderm ${ }^{\circledR}$ dressing to cover all valves and tubing junction sites to prevent tampering. The IV lines are inspected daily, and ID providers were contacted to discuss any patients suspicious of tampering with their lines to discuss next appropriate steps, either readmission or transition to oral antibiotics. Half of our patients were switched to oral therapy during their course, consistent with current literature. ${ }^{12,14}$

Traditionally, homeless patients requiring ongoing parenteral therapy have remained inpatients for the duration of their course. Feigal and colleagues evaluated the connection between homelessness and inpatient discharge delays for placement over a 6-month period in 2009 at an urban hospital. ${ }^{15}$ They found homeless patients awaiting placement had an increased median LOS of 26 days, compared to housed individuals with 14 days. Homeless patients without a psychiatric disorder had a delay in discharge $60 \%$ longer compared to those with housing, with data adjusted for multiple variables. The cause for delay in discharge in homeless patients was found in those awaiting group home or nursing facility placement, in $50 \%$ of cases, whereas delay for chemical dependency program was in $17 \%$ of cases, and other local treatment center in $12 \%$ of cases.

Medical respite programs are gaining in popularity in the United States since they began in the mid1980s. ${ }^{16}$ A review by Doran and colleagues found medical respite can result in cost avoidance for hospitals by limiting inpatient days and readmissions. ${ }^{17}$ Medical respite can also help engage patients in follow-up care and assist with housing placement. Many programs promote "safe" IDU practices and offer referrals for rehabilitation programs, both of which are programs that are not available in most hospitals. Medical respite may continue to be a site of OPAT expansion, as there is continued pressure to discharge nonacute patients from the hospital. Moving forward, it may be beneficial for hospitals, public health departments, and communities to support these programs, which can assist with close monitoring of homeless patients receiving OPAT.

There were several limitations in our study. This was a retrospective observational study with a small patient population comprised of a high prevalence of current and remote IDU. The single center study makes it difficult to generalize to other settings. In addition, there were no comparative data with historical controls, making it difficult to perform comparative analysis.

OPAT is effective for many patients, and it is optimal to utilize ID consultation to determine appropriate candidacy, ${ }^{3-5}$ particularly among IDU. OPAT can be successful in a closely monitored medical respite setting for homeless patients with the help of a multidisciplinary team. Medical respite OPAT can decrease inpatient stays in patients who would otherwise require long hospitalizations, resulting in overall cost savings, and may lead to improved patient satisfaction. Future research linking other outcomes of medical respite OPAT, including substance-dependence treatment and transition to housing, is warranted.

\section{Acknowledgements}

The authors thank the staff at the Harborview Medical Center Infectious Disease Clinic and at Edward Thomas House Medical Respite for their help in this study.

Disclosures: Presented at the oral abstract session Clinical Practice Issues-OPAT in Diverse Populations, IDWeek, October 8-12, 2014, Philadelphia, Pennsylvania. The authors report no conflicts of interest.

\section{References}

1. Chary A, Tice AD, Liedtke LA, et al. Experience of infectious diseases consultants with outpatient parenteral antimicrobial therapy: results of an emerging infections network survey. Clin Infect Dis. 2006;43: 1290-1295.

2. Corwin P, Toop L, McGeoch G, et al. Randomized controlled trial of intravenous antibiotic therapy for cellulitis at home compared with hospital. BMJ. 2005;330:129.

3. Tice AD, Rehm SJ, Dalovisio JR, et al. Practice guidelines for outpatient parenteral antimicrobial therapy. IDSA guidelines. Clin Infect Dis. 2004;38:1651-1672.

4. Conant MM, Erdman SM, Osterholzer D. Mandatory infectious diseases approval of outpatient parenteral antimicrobial therapy (OPAT): clinical and economic outcomes of averted cases. I Antimicrob Chemother. 2014;10:1093-1099.

5. Muldoon EG, Snydman DR, Penland EC, Allison GM. Are we ready for an outpatient parenteral antimicrobial therapy bundle? A critical appraisal of the evidence. Clin Infect Dis. 2013;57:419-424.

6. Marra CA, Frighetto L, Goodfellow AF, et al. Willingness to pay to access patient preferences for therapy in a Canadian setting. BMC Health Serv Res. 2005;5:43.

7. UW Medicine. Respite program at Jefferson Terrace (Edward Thomas House). University of Washington website. Available at: http://www. uwmedicine.org/locations/respite-program-jefferson-terrace. Accessed October 1, 2015.

8. Boston Healthcare for the Homeless Program. Medical respite care at the Barbara McInnis House. Available at: http://www.bhchp.org/ medical-respite-care. Accessed October 1, 2015.

9. San Francisco Department of Public Health. Medical Respite and Sobering Center. Available at: https://www.sfdph.org/dph/comupg/ oprograms/HUH/medrespite.asp. Accessed October 1, 2015.

10. Harris PA, Taylor R, Thielke R, Payne J, Gonzalez N, Conde JG. Research electronic data capture (REDCap)—a metadata-driven methodology and workflow process for providing translational research informatics support. J Biomed Inform. 2009;42:377-381.

11. Barr DA, Semple L, Seaton RA. Outpatient parenteral antimicrobial therapy (OPAT) in a teaching hospital-based practice: a retrospective cohort study describing experience and evolution over 10 years. Int J Antimicro Agents. 2012;39:407-413.

12. Allison GM, Muldoon EG, Kent DM, et al. Prediction model for 30-day hospital readmissions among patients discharged receiving outpatient parenteral antibiotic therapy. Clin Infect Dis. 2014;58: $812-819$.

13. Ho J, Archuleta S, Sulaiman Z, Fisher D. Safe and successful treatment of intravenous drug users with a peripherally inserted central catheter in an outpatient parenteral antibiotic treatment service. J Antimicrob Chemother. 2010;65:2641-2644. 
14. Gilchrist M, Seaton RA. Outpatient parenteral antimicrobial therapy and antimicrobial stewardship: challenges and checklists. J Antimicrob Chemother. 2015;70:965-970.

15. Feigal J, Park B, Bramante C, Nordgaard C, Menk J, Song J. Homelessness and discharge delays from an urban safety net hospital. Public Health. 2014;128:1033-1035.
16. Zerger S, Doblin B, Thompson L. Medical respite care for homeless people: a growing national phenomenon. J Health Care Poor Underserved. 2009;20:36-41.

17. Doran KM, Ragins KT, Gross CP, Zerger S. Medical respite programs for homeless patients: a systematic review. J Health Care Poor Underserved. 2013;24:499-524. 